\title{
Facile and green preparation of magnetite/zeolite nanocomposites for energy application in a single-step procedure
}

\begin{abstract}
This paper presents a green, facile and rapid method to prepare magnetite/zeolitenanocomposites (NCs) in one step procedure at ambient temperature. The powder X-ray diffraction (PXRD) pattern of iron oxide nanoparticles (NPs) with the sole zeolite showed the broadening of zeolite peaks attributed to the incorporation of Fe3O4. Field-emission scanning electron microscopy (FESEM) analysis depicted that the Fe3O4-NPs were formed on the surface of porous zeolite framework. Transmission electron microscopy (TEM) analysis displayed the Fe3O4 nanoparticles (NPs) were mostly in spherical shape with a mean diameter and standard deviation of $2.40 \pm 0.41 \mathrm{~nm}$. The selected-area electron diffraction (SAED) pattern confirmed the presence of cubic $\mathrm{Fe} 3 \mathrm{O} 4$ phase. The vibrating sample magnetometer (VSM) results indicated the as-synthesized sample has a saturation magnetization of around $6.52 \mathrm{emu} \mathrm{g}-1$. The magnetite/zeolite-NCs can be considered as a low-cost alternative catalyst for oxygen reduction reaction (ORR) process. The electrochemical measurement showed that the performance of magnetite/zeolite-NCs towards the ORR increased as the scan rate increased from $20 \mathrm{mV} \mathrm{s}-1$ to $500 \mathrm{mV} \mathrm{s}-1$. The ORR is a diffusion-controlled process in the alkaline medium.
\end{abstract}

Keyword: Green synthesis; Fe3O4 nanoparticles; Zeolite; Nanocomposites; Oxygen reduction reaction 